\title{
Horta vertical com garrafas PET para a construção de consciência ambiental de
}

\section{estudantes}

\author{
Vertical vegetable garden with pet bottles for students environmental awareness construction \\ Casa vertical con botellas pet para la construcción de la conciencia ambiental de estudiantes
}

Recebido: 08/01/2021 | Revisado: 11/01/2021 | Aceito: 16/01/2021 | Publicado: 19/01/2021

\author{
Adrielle de Souza Santos \\ ORCID: https://orcid.org/0000-0002-1412-5678 \\ Instituto Federal de Educação, Ciência e Tecnologia Goiano, Brasil \\ E-mail: adriellesantos@gmail.com \\ Thaís Silva de Souza \\ ORCID: https://orcid.org/0000-0002-9232-2001 \\ Instituto Federal de Educação, Ciência e Tecnologia Goiano, Brasil \\ E-mail: thaisbioif06@gmail.com \\ Maisa Souza Lemes \\ ORCID: https://orcid.org/0000-0001-5522-4149 \\ Instituto Federal de Educação, Ciência e Tecnologia Goiano, Brasil \\ E-mail: maisa31121998@gmail.com \\ Wanderson Siqueira Teles \\ ORCID: https://orcid.org/0000-0002-1136-6919 \\ Instituto Federal de Educação, Ciência e Tecnologia Goiano, Brasil \\ E-mail: wandersonteles123@gmail.com \\ Caroliny Fatima Chaves da Paixão \\ ORCID: https://orcid.org/0000-0002-6445-2554 \\ Instituto Federal de Educação, Ciência e Tecnologia Goiano, Brasil \\ E-mail: carolinyfcpaixao@gmail.com \\ André Luis da Silva Castro \\ ORCID: https://orcid.org/0000-0001-9455-1169 \\ Instituto Federal de Educação, Ciência e Tecnologia Goiano, Brasil \\ E-mail: andrelscastro@gmail.com
}

\begin{abstract}
Resumo
Este estudo tem por objetivo relatar uma pesquisa e prática ambiental realizadas por meio da construção de uma horta vertical com garrafas PET com estudantes do $1^{\circ}$ ano do Ensino Médio, no município de Urutaí-GO, sudeste do estado de Goiás. Para isso, inicialmente foi aplicado um pré-teste aos estudantes para avaliar o nível de interesse e preocupação em relação a alimentação saudável, produtos orgânicos, sustentabilidade e hortas orgânicas. Após o preenchimento do pré-teste foi ministrada uma aula sobre a temática ambiental e nutricional e confeccionada uma horta vertical, com garrafas PET, com a participação dos estudantes. Foram plantadas mudas de alface, cebolinha e hortelã. Após as atividades foi aplicado um pós-teste. Os resultados indicam que os estudantes se mostraram mais preocupados com a alimentação saudável e questões ambientais após a aula explicativa e a prática de construir a horta vertical. Conclui-se que a atividade com a horta vertical com garrafas PET contribuiu para o aumento de interesse dos estudantes por questões ambientais e nutricionais, o que a torna um bom instrumento para a construção de consciência ambiental de jovens.
\end{abstract}

Palavras-chave: Educação ambiental; Sustentabilidade; Horta vertical; Ensino.

\begin{abstract}
This study aims to report a research and environmental practice carried out through the construction of a vertical vegetable garden with PET bottles with students of the first year of high school, in the municipality of Urutaí-GO, southeast of the state of Goiás. A pre-test was applied to the students to assess the level of interest and concern regarding healthy eating, organic products, sustainability and organic vegetable gardens. After completing the pre-test, a class on environmental and nutritional issues was given and a vertical vegetable garden with PET bottles was made, with the participation of the students. Seedlings of lettuce, chives and mint were planted. After the activities, a posttest was applied. The results indicate that the students were more concerned with healthy eating and environmental issues after the explanatory class and the practice of building the vertical vegetable garden. It is concluded that the vertical vegetable garden contributes to the increase of interest of students for environmental and nutritional issues, which makes it a good instrument for building environmental awareness of young people.
\end{abstract}

Keywords: Environmental education; Sustainability; Vertical vegetable Garden; Teaching. 


\section{Resumen}

Este estudio tiene como objetivo reportar una investigación y práctica ambiental realizada a través de la construcción de un jardín vertical con botellas de PET con estudiantes de $1^{\circ}$ año de secundaria, en el municipio de Urutaí-GO, al sureste del estado de Goiás. Se aplicó una prueba previa a los estudiantes para evaluar el nivel de interés y preocupación en relación a la alimentación saludable, productos orgánicos, sostenibilidad y huertos orgánicos. Luego de completar el pre-test, se dio una clase sobre el tema ambiental y nutricional y se realizó un jardín vertical con botellas PET, con la participación de los estudiantes. Se sembraron plantones de lechuga, cebollino y menta. Después de las actividades, se aplicó una prueba posterior. Los resultados indican que los estudiantes estaban más preocupados por la alimentación saludable y los problemas ambientales después de la clase explicativa y la práctica de construir el jardín vertical. Se concluye que la actividad con el jardín vertical con botellas de PET contribuyó al aumento del interés de los estudiantes por los temas ambientales y nutricionales, lo que lo convierte en un buen instrumento para la construcción de conciencia ambiental entre los jóvenes.

Palabras clave: Educación ambiental; Sostenibilidad; Jardín vertical; Ensenãnza.

\section{Introdução}

O crescimento populacional demanda o aumento da produção agropecuária e industrial, causando profundas transformações socioambientais (Ismael et al., 2016). Juntamente com o aumento populacional ocorreram transformações nos padrões de vida da sociedade, que se tornou consumista e adepta ao descartável, gerando então uma problemática mundial: a produção excessiva de resíduos sólidos e o seu descarte no meio ambiente, que causam graves impactos em todo o ecossistema (Marchi, 2018; Ismael et al., 2016; Cândido et al., 2017).

O descarte inadequado do lixo pode causar a contaminação dos lençóis freáticos e corpos d'água, assoreamento de rios e lagos, proliferação de vetores e transmissores de doenças, além de enchentes (Bravo, 2008). Em virtude dos impactos gerados pelo acúmulo de resíduos sólidos no meio ambiente torna-se urgentes ações mitigatórias desses impactos. A adoção do princípio dos 3 R's (reduzir, reciclar e reutilizar) é um caminho para a solução dos problemas relacionados à produção de lixo, visando o estabelecimento de padrões de consumo sustentáveis (Marchi, 2018).

Um dos materiais produzidos e descartados em grandes volumes é o PET (politereftalato de etileno), utilizado principalmente em embalagens de refrigerantes, água, sucos, óleos comestíveis e diversos outros produtos, devido à sua grande resistência e durabilidade (Almeida et.al., 2013). O PET vem sendo utilizado desde 1990 e, devido ao seu descarte inadequado e à sua persistência, que pode ultrapassar 400 anos para sua biodegradação, transformou-se em um sério problema ambiental mundial (Almeida et.al., 2013).

Apesar dos avanços da legislação, nem todos os municípios brasileiros são beneficiados com áreas para coleta seletiva (Santagueda et al., 2020). Segundo (Conke \& Nascimento, 2018) apenas $41 \%$ dos municípios estariam sendo privilegiados com esses serviços. Sendo assim, a reutilização de alguns materiais passa a ser uma ótima alternativa, e um dos objetos mais agressores ao meio ambiente são as garrafas PET. Mas com as embalagens são possíveis a confecção de diversos produtos para decoração, vasos, puffs, porta objetos, luminárias e outras centenas de coisas. (Pinheiro et al., 2020).

Nesse contexto, é importante o desenvolvimento de atividades na sociedade em prol do consumo consciente e de estratégias para minimizar os impactos causados pelos resíduos sólidos. O desenvolvimento de práticas de Educação Ambiental que visam a transformação dos resíduos em objetos utilizáveis no dia a dia é essencial, além de contribuir para a formação de cidadãos críticos e no desenvolvendo habilidades nos participantes (Oliveira \& Moura, 2017).

O presente trabalho tem por objetivo relatar uma pesquisa e prática ambiental realizadas por meio da construção de uma horta vertical com garrafas PET com estudantes do Ensino Médio no município de Urutaí, sudeste do Estado de Goiás.

\section{Metodologia}

A metodologia aplicada tem caráter de pesquisa-ação participativa, a qual foi desenvolvida por Michel Thiollent, sendo destinada a resolução de problemas, com a participação dos envolvidos de forma ativa buscando soluções para o 
problema trabalhado e durante o percurso compreendendo o que se está fazendo e o porquê (Pereira et al., 2018). Pereira et al., (2018) apontam ainda que as escolas são ambientes favoráveis para a aplicação da pesquisa-ação, pois os problemas sejam educacionais, de infraestrutura, ou até mesmo sociais nem sempre é resolvido de forma individual, necessita da participação de todos da unidade escolar. O método também é de forma quantitativo, o qual utiliza-se de uma coleta de dados, com valores números que posteriormente são analisados gerando dados estatísticos (Pereira et al., 2018).

As atividades foram desenvolvidas no Colégio Estadual Dr. Vasco dos Reis Gonçalves, município de Urutaí, sudeste do estado de Goiás, no período de 4 de abril a 26 de maio de 2017. Participaram da pesquisa 16 estudantes do $1^{\circ}$ ano do Ensino Médio, do turno matutino, com idade média de 15 anos.

Inicialmente, foi aplicado um pré-teste aos alunos para avaliar o nível de preocupação e interesse dos estudantes em relação a alimentação saudável, produtos orgânicos, sustentabilidade e hortas orgânicas. O questionário continha perguntas para preenchimento em uma escala de 1 a 5 , sendo 1 muito desinteressado/despreocupado e 5 muito interessado/preocupado em relação aos aspectos abordados.

Após o preenchimento do pré-teste foi ministrada uma aula, com uso de data-show e quadro negro, abordando conceitos de sustentabilidade, importância da redução do consumo, reutilização de materiais, incentivo ao consumo de alimentos saudáveis e hortas orgânicas, com duração de 2 horas. Foram abordados ainda os critérios técnicos importantes para a construção de uma horta, como local de instalação, tipo de substratos, espécies de hortaliças a serem cultivadas, luminosidade, cuidados hídricos etc.

Após a aula, os materiais necessários para a confecção da horta vertical foram levados para a escola: 24 garrafas PET de refrigerante de 2,0L e 2,5L (vazias e limpas), arame liso, tesoura grande, alicate de ponta fina, alicate de ponta grossa, faca, substratos para o plantio (húmus de minhoca, terra, carvão moído, pó de rocha, fosfato de rocha, cinza vegetal e calcário dolomítico).

Os alunos foram orientados sobre como confeccionar a horta vertical e participaram de cada etapa, de modo que era possível sanar dúvidas, promover interação e consolidar o conhecimento a respeito da construção da mesma. A Figura 1 mostra o resultado final da horta vertical construída pelos alunos com o auxílio dos realizadores do projeto.

Figura 1. Horta vertical construída com garrafas PET recicladas no Colégio Estadual Dr. Vasco dos Reis Gonçalves, UrutaíGO, pelos alunos do $1^{\circ}$ ano do Ensino Médio. a) Vista geral da horta vertical; b) Detalhe da horta vertical construída, com mudas de salsa e alface.

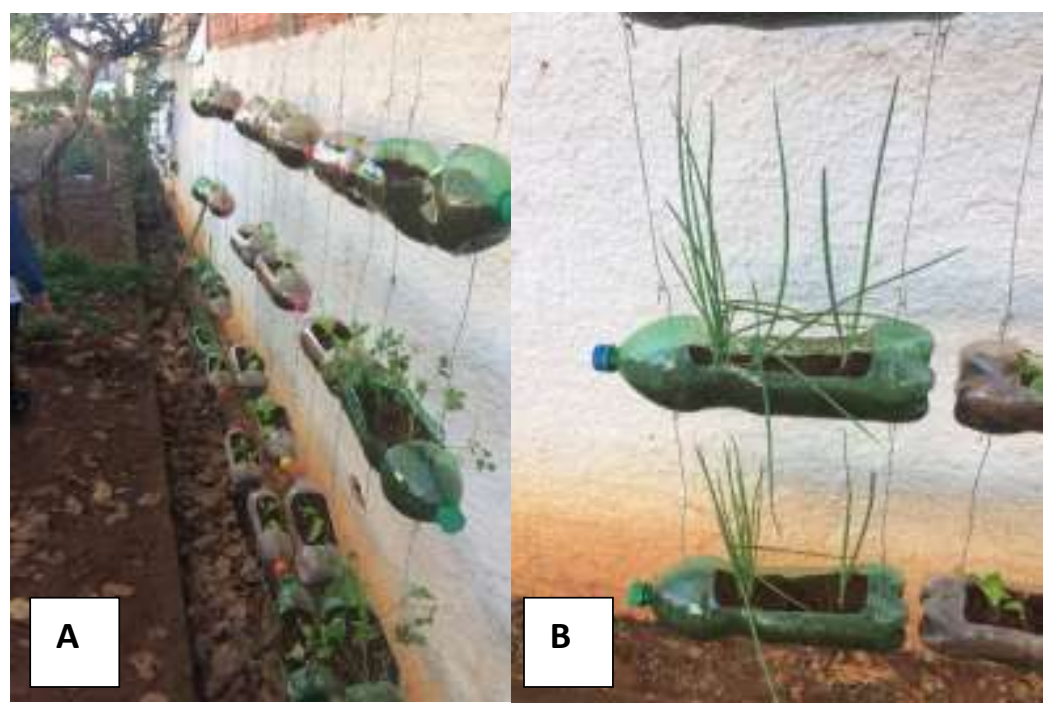

Fonte: Autores. 
Para o preparo da horta vertical foram adotados os seguintes passos: (i) corte das garrafas (removendo um retângulo nas laterais de aproximadamente $18 \times 10 \mathrm{~cm}$ para garrafas de 2,0L e $21 \times 11 \mathrm{~cm}$ para garrafas de 2,5L) e perfuração das mesmas para passagem do arame; (ii) mistura dos substratos e transferência dos mesmos para as garrafas; (iii) fixação das garrafas em uma das paredes da escola; (iv) plantio das mudas; (v) primeira rega das plantas. Cada aluno ficou responsável pelo plantio e manutenção de duas mudas, com distância de $7 \mathrm{~cm}$ entre elas.

As espécies escolhidas para plantio foram: alface crespa (Lactuca sativa), salsinha (Petroselinum crispum), cebolinha (Allium schoenoprasum) e hortelã (Mentha piperita), em função da facilidade de cultivo em garrafas pet e a possibilidade de uso para a culinária. Os alunos elaboraram uma escala para rega da horta diariamente e um grupo no WhatsApp foi criado para comunicação e acompanhamento para os cuidados diários com a horta.

Após a confecção e manutenção da horta vertical, o mesmo questionário foi aplicado (pós-teste), visando avaliar possíveis mudanças de preocupação e interesse dos estudantes em relação à horta e aspectos ambientais.

\section{Resultados e Discussão}

Foram confeccionadas 24 unidades de hortas verticais (24 garrafas PET), nas quais foram cultivadas 49 mudas, sendo 33 de alface (Lactuca sativa), 2 de hortelã (Mentha piperita), 6 de salsinha (Petroselinum crispume) e 8 de cebolinha (Allium schoenoprasum).

O desenvolvimento da atividade ocorreu sem grandes dificuldades, com baixo custo e grande envolvimento e participação dos estudantes, indicando viabilidade da mesma em ações de Educação Ambiental. Além disso, os questionários pré e pós-teste permitiram quantificar o interesse e preocupação dos estudantes. A Figura 2 mostra o nível de interesse dos alunos a respeito do assunto, antes da realização do projeto e após a realização do mesmo. Dos seis aspectos questionados, houve aumento de interesse no pós-teste em quatro aspectos (interesse sobre a procedência das hortaliças, em cultivar horta na escola, aprender técnicas de cultivo e reutilizar objetos). Além disso, os estudantes mostraram-se mais preocupados após a realização da aula e a participação na construção da horta vertical PET, conforme ilustra a Figura 3.

Böhm et al. (2017) relatam que muitas pessoas têm noção do que seja um alimento saudável, entretanto, não têm consciência dos riscos de uma alimentação com resíduos de agroquímicos. Stefano et al. (2007) ressaltam ainda que o valor nutricional e a qualidade dos alimentos orgânicos são maiores quando comparados a alimentos não orgânicos, o que proporciona uma melhor absorção de nutrientes, sendo mais vantajoso para a saúde.

Em contrapartida, em relação a cultivar horta em casa, não houve diferença de interesse dos estudantes entre pré-teste e pós-teste, como mostra a Figura 2. Isso pode estar relacionado ao fato de $81,25 \%$ ( $n=13$ ) dos estudantes já terem tido experiência com horta em casa, sendo que 62,5\% ainda possuem a mesma. Os outros 18,75\% (n=3) que não cultivam horta em casa relataram falta de conhecimento e principalmente preguiça. É importante salientar que 100\% ( $\mathrm{n}=16)$ dos estudantes responderam que tinham condições de cultivar horta em casa e 93,75\% (n=15) dispunham de espaço físico para isso. 
Figura 2. Interesse dos estudantes do $1^{\circ}$ ano do Ensino Médio $(n=16)$, do Colégio Estadual Dr. Vasco dos Reis Gonçalves, Urutaí-GO, sobre horta, hortaliças e reciclar ou reutilizar objetos. Média \pm desvio padrão dos escores obtidos, sendo cinco como muito interessado e 0 como nada interessado, no pré-teste e pós-teste (após realização das atividades).

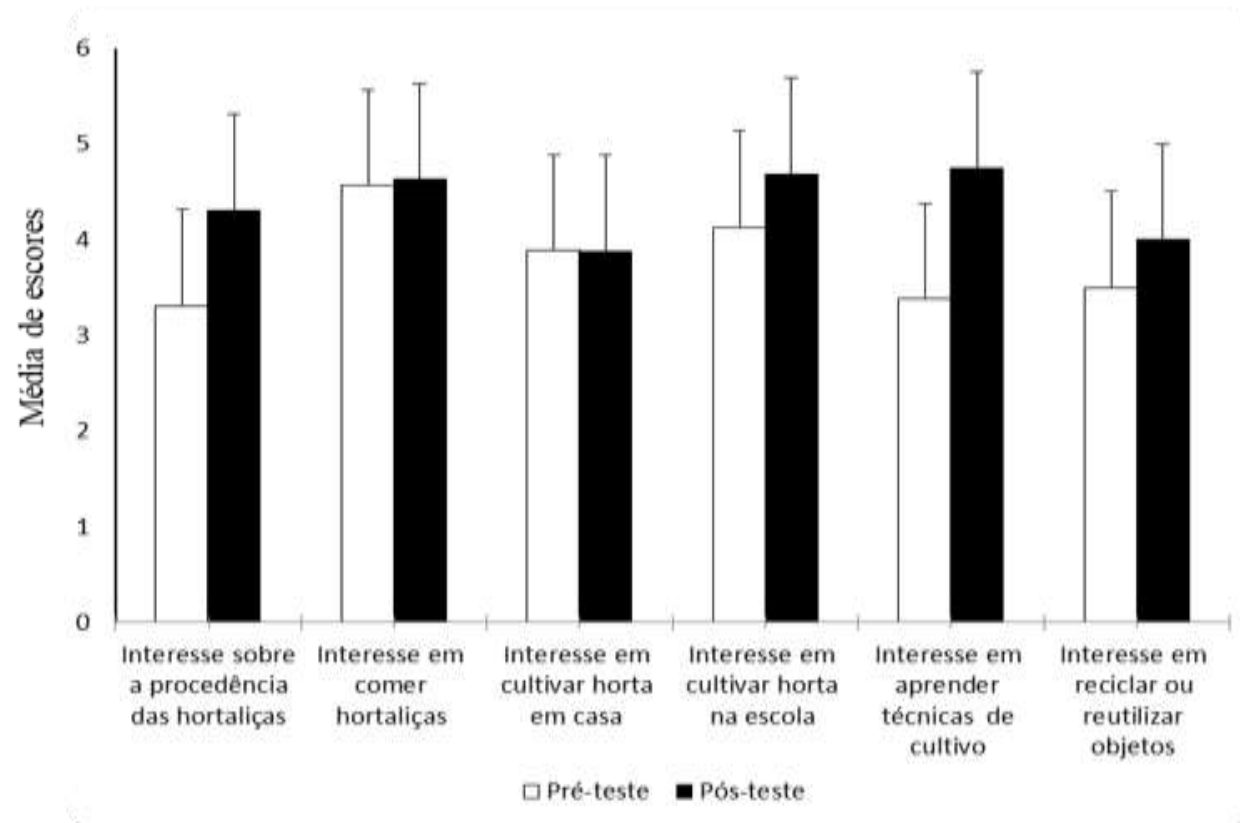

Fonte: Autores.

Figura 3. Preocupação de alunos do $1^{\circ}$ ano do Ensino Médio $(n=16)$, do Colégio Estadual Dr. Vasco dos Reis Gonçalves, Urutaí-GO, em relação ao consumo de hortaliças com agrotóxicos, a ingerir hortaliças diariamente e ao descarte inadequado de lixo. Média \pm desvio padrão dos escores obtidos, sendo cinco como muito interessado e 0 como nada interessado, no pré-teste e pós-teste (após realização das atividades).

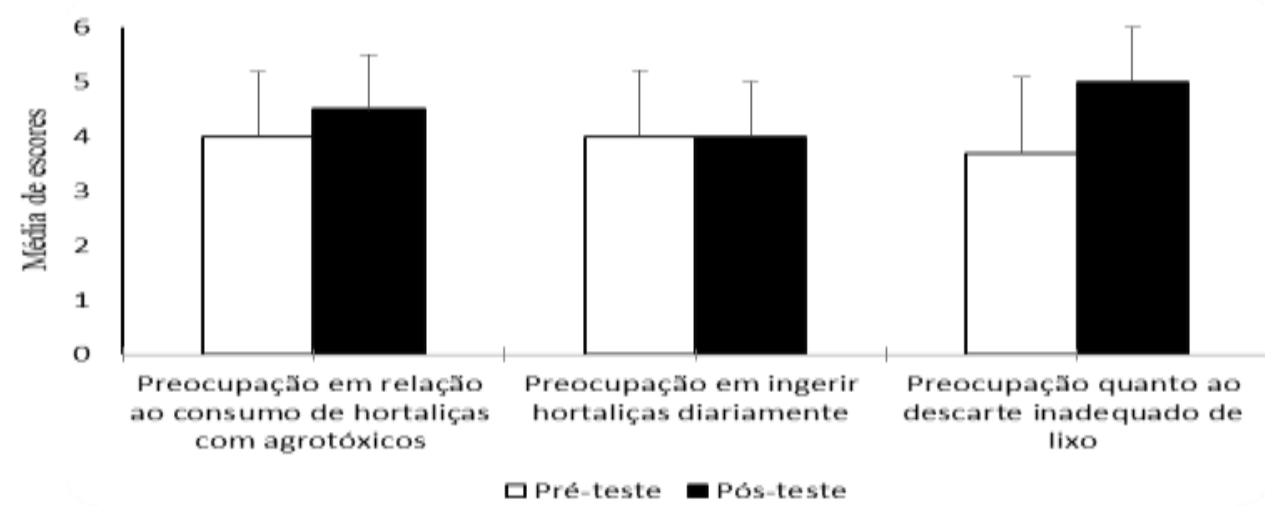

Fonte: Autores. 
O pós-teste indica aumento do interesse dos estudantes para reciclagem e reutilização de materiais. Sobre esse aspecto, Marodin e Morais (2004) corroboram os resultados, revelando que educação sobre reciclagem faz com que o lixo seja visto de outra maneira, não como não como um destino final, mas como o início de um ciclo no qual é possível a conservação do meio ambiente (Marodin \& Morais, 2004).

De acordo com Oliveira e Moura. (2017), as abordagens didáticas em que o aluno é o centro das atenções contribuem diretamente para o aumento do interesse dos estudantes, tornando-as mais atrativas. Alguns trabalhos apontam que atividades como o cultivo da horta vertical no espaço escolar são interessantes por torna-se um laboratório de possibilidades, promovendo aos alunos aulas diferenciadas e possibilitando a compreensão de um consumo mais sustentável. (Fernandes et al., 2020; Oliveira et al., 2018). Outros aspectos positivos que podem ser destacados em atividades semelhantes são o embelezamento do local, o que resulta na diminuição de poluição visual para quem utiliza o espaço e o despertar de interesse pelos alimentos cultivados nas hortas (Melo et al., 2016).

Sobre a preocupação dos estudantes, não houve alteração no pós-teste em relação a consumir hortaliças diariamente e houve aumento de preocupação em consumir hortaliças com agrotóxicos e com o descarte inadequado do lixo, conforme ilustra a Figura 3. As hortaliças constituem um dos grupos alimentares com maior rejeição entre os adolescentes (Iglesias et al.,2008; Kotecha et al., 2013). Contudo, o impacto da baixa ingestão de hortaliças pode associar-se a diminuição de micronutrientes causando retardo no desenvolvimento, diminuição da capacidade de aprendizagem, entre outros (Ferreira et al.,2007; Finger et al.,2015; Stea \& Torstveit, 2014). Em relação à preocupação com o consumo de hortaliças com agrotóxicos, de Melo Moura (2020) alerta que o consumo de alimentos com agrotóxicos pode contribuir para o desenvolvimento de várias doenças, como câncer o que ressalta a importância do resultado obtido.

Quanto a preocupação com o descarte inadequado do lixo, o aumento de preocupação dos estudantes é um importante resultado, uma vez que estudantes tendem a não se preocupar com a destinação final do lixo (Costa, 2018). Böhm et al. (2017) afirmam que, em uma sociedade que prioriza o consumo não existe a preocupação com a produção e descarte dos produtos, é de suma importância a promoção de debates guiados pela educação ambiental nas escolas para repensar esse comportamento. Ao final das atividades, o pós-teste revelou que $100 \%$ dos estudantes avaliaram-nas como ótima, indicando excelente aceitação dos mesmos sobre esse aspecto. Da Silva (2019) ressalta que uso de metodologias de ensino diversificadas, como práticas associadas à teoria, tornam a construção de conhecimento mais clara, atrativa e de maior qualidade.

De acordo com Souza, Holanda e Castro (2016), atividades de Educação Ambiental diferenciadas devem ser estimuladas, uma vez que as práticas tradicionais desenvolvidas não propiciam apropriação crítica, e, portanto, não promovem uma reflexão sobre a relação entre ser humano e meio ambiente. No caminho de efetivar a Educação Ambiental, cabe ao professor a tarefa de guiar os educandos e criar situações para que de forma construtiva desenvolvam competências e habilidades, que resultam na consciência da necessidade de conservação ambiental (Aguiar et al., 2017). Nesse sentido, Cribb (2010) e Freixo (2009) reforçam que comportamentos ambientalmente corretos como, por exemplo, a separação do lixo e reutilização de materiais, devem ser aprendidos de forma prática, no cotidiano escolar, contribuindo para a formação de cidadãos responsáveis.

\section{Conclusão}

As atividades desenvolvidas na confecção da horta vertical com garrafas PET mostram-se de baixo custo, viáveis, participativas e contribuem para o aumento de interesse dos estudantes por questões ambientais e nutricionais, o que as tornam um bom instrumento para a construção de consciência ambiental de jovens. Deste, é importante que atividades como essa sejam replicadas nas escolas, para tanto é necessário a elaboração e o desenvolvimento de novas propostas de intervenção semelhantes, como por exemplo, a implementação de novas variedades de hortaliças na horta, uma futura aula prática de 
culinária utilizando os produtos da horta e até mesmo uma feira, para mostrar os produtos a toda a comunidade e aos pais. Estas atividades de caráter ativo, vem contribuir de forma prática com a formação de cidadãos conscientes.

\section{Referências}

Aguiar, P. C. B., Neto, R. F. C., Bruno, N. L., \& Profice, C. C. (2017). Da teoria à prática em educação ambiental. Revista Gestão \& Sustentabilidade Ambiental, 6(2), 111-132.

Almeida, I. S., Costa, I. D., Ribeiro, M. M., Heinrich, M., Moreira, Q., Araujo, P. J., \& Leite, M. S. (2013). Reciclagem de garrafas PET para fabricação de telhas. Caderno de Graduação-Ciências Exatas e Tecnológicas-UNIT-SERGIPE, 1(3), 83-90.

Bohm, F. Z., Böhm, P. A. F., Rodrigues, I. C., \& Júnior, M. P. S. (2018). Utilização de hortas orgânicas como ferramenta para Educação Ambiental. Luminária, 19(01).

Bravo, T. L., Peçanha, A. L., Werner, E. T., \& Santos, A. A. O. (2018). Educação ambiental e percepção da implantação de coleta seletiva de lixo urbano em de alegre, es. Revista Gestão \& Sustentabilidade Ambiental, 7(1), 375-396.

Cândido, J., Souza, P. A., Neres, N. G. C., Gonçalves, D. S., \& Souza, P. B. (2017). Diagnóstico ambiental e análise temporal dos impactos ambientais causados por um depósito de resíduos sólidos no município de cariri do Tocantins-To. Nucleus, 14(1).

Coelho, D. E. P., \& Bógus, C. M. (2016). Vivências de plantar e comer: a horta escolar como prática educativa, sob a perspectiva dos educadores. Saúde \& Sociedade, São Paulo, 25(3),761-771.

Conke, L. S., \& Nascimento, E. P. D. (2018). A coleta seletiva nas pesquisas brasileiras: uma avaliação metodológica. urbe. Revista Brasileira de Gestão Urbana, 10(1), 199-212.

Corrêa, R. F. M. (2010). Reutilização de garrafas pet para produção de móveis e desenvolvimento socioambiental. Interciência \& Sociedade, 1(1), 129-135.

Costa, R. L. (2018)."Conscientização dos alunos do $1^{\circ}$ ano do ensino médio da destinação do RSU.", $30 \mathrm{f.}$

Da Silva, T. D. S. G. (2019). Ensino de ciências e experimentação nos anos iniciais: da teoria à prática. Pró-Discente, 25(1).

Fernandes, R. O., Zajac, M. A. L., Ribeiro, A. P., \& do Nascimento, A. P. B. (2020). Avaliação de ações de práticas de sustentabilidade em escola municipal de ensino infantil na zona sul da cidade de São Paulo-SP. Interfaces Científicas-Educação, 8(3), 393-407.

Ferreira, A., Chiara, V. L., \& Kuschnir, M. C. C. (2007). Alimentação saudável na adolescência: consumo de frutas e hortaliças entre adolescentes brasileiros. Adolescencia e Saude, 4(2), 48-52.

Ferreira, E. A. (2012). Horta vertical na escola. Monografia de Especialização (Ensino de Ciências). Universidade Tecnológica Federal do Paraná, Medianeira, $31 \mathrm{f}$.

Finger, J. D., Varnaccia, G., Tylleskär, T., Lampert, T., \& Mensink, G. B. M. (2015) Dietary behaviour and parental socioeconomic position among adolescents: the German Health Interview and Examination Survey for Children and Adolescents 2003-2006 (KiGGS). BMC Public Health, 15(498), 1-13.

Iglesias-Gutiérrez, E., García-Rovés, P. M., García, A., \& Patterson, A. M. (2008). Food preferences do not influence adolescent high-level athletes' dietary intake. Appetite. 50, (2-3), 536-43.

Ismael, D. A. M. (2016). Proposta de um plano de recuperação de área degradada para o "lixão" de Pombal-PB.

Kotecha, P. V., Patel, S. V., Baxi, R. K., Mazumdar, V. S., Shobha, M., Mehta, K. G., \& Ekta, M. (2013). Dietary pattern of schoolgoing adolescents in urban Baroda, India. Journal of health, population, and nutrition, 31(4), 490.

Lima, A. S. D., Duarte, K., \& Araújo, E. P. (2014). Confecção de uma horta vertical utilizando garrafa PET na Escola Estadual Clóvis Pedrosa, CabaceirasPB. In Anais do V Congresso Brasileiro de Gestão Ambiental. Belo Horizonte: IBEAS (pp. 1-6).

Marchi, C. M. D. F. (2018). Cenário mundial dos resíduos sólidos e o comportamento corporativo brasileiro frente à logística reversa. Perspectivas em Gestão \& Conhecimento, 1(2), 118-135.

Melo Moura, C. C., Pires, C. V., Madeira, A. P. C., \& Macedo, M. C. C. (2020). Perfil de consumidores de alimentos orgânicos. Research, Society and Development, 9(9), e257997395-e257997395.

Melo, E. F. R. Q., Giglioli, A., \& Pires, T. B. (2016) Educação ambiental e reciclagem de materiais associados ao paisagismo produtivo. Anais da V Jornada de Extensión del Mercosur. Passo Fundo: UPF, 1-9.

Mucelin, C. A., \& Bellini, M. (2008). Lixo e impactos ambientais perceptíveis no ecossistema urbano. Sociedade \& natureza, 20(1), $111-124$.

Oliveira, F., Pereira, E., \& Júnior, A. P. (2018). Horta escolar, Educação Ambiental e a interdisciplinaridade. Revista Brasileira de Educação Ambiental (RevBEA), 13(2), 10-31.

Oliveira, L. A., \& Moura, J. D. P. (2017). Educação Ambiental por meio da reutilização de resíduos e construção de jogos. Revista Brasileira de Educação Ambiental, 12(2), 127-135.

Pereira, A. S., Shitsuka, D. M., Parreira, F. J., \& Shitsuka, R. (2018). Metodologia da pesquisa Científica. E-book. 1. ed. Santa Maria. RS: UFSM, NTE. https://repositorio.ufsm.br/bitstream/handle/1/15824/Lic_Computacao_Metodologia-Pesquisa-Cientifica.pdf?sequence=1. 
Research, Society and Development, v. 10, n. 1, e39510111804, 2021

(CC BY 4.0) | ISSN 2525-3409 | DOI: http://dx.doi.org/10.33448/rsd-v10i1.11804

Santagueda, V. M. P., da Silva Cantalice, A., da Silva, A. B., \& Mafort, M. E. (2020). Comportamento sustentável: promoção

Souza, R. F., Holanda, A. R., \& Castro, O. M. (2016). Abordagens de ensino de educação ambiental no ensino médio da ETSC/UFCG. Anais Congresso Nacional de Educação: Realize.

Stea, T. H., \& Torstveit, M. K. (2014). Association of lifestyle habits and academic achievement in Norwegian adolescents: a cross-sectional study. BMC Public Health. 14, (829), 2-8.

Stefano, N., Godoy, L. P., \& Ruppenthal, J. E. (2007). Uma análise reflexiva do comportamento dos consumidores de produtos orgânicos. Anais XIV Simpósio em Engenharia de Produção - SIMPEP. Bauru, São Paulo.

Vieira, L. A., dos Santos, M. M. C., Vieira, T. A., dos Santos Oliveira, J., \& da Silva Alves, H. (2020). Alimentação saudável sob o olhar da Educação Ambiental. Research, Society and Development, 9(12), e45691211237-e45691211237. 